\title{
Summary of Material
}

\section{Romans}

Complete: 51; 54; 58; 61; 75; 76 (lac. 8:21-33, 11:15-25); 77; 78 (lac. 1:1-3:19); 88; 89; 135; AMst; PEL; RUF

\section{Partial:}

1: 84 (v. 13); 86; AU (vv. 1-7, 11, 18, 21, 24, 28-29, 32); CAr (vv. 1-3, 8, 18, 24); TES (vV. 25-26); SPM (vv. 22-26); SPE (vv. 16-32)

2: 86; AU (vV. 1, 5, 15, 29); CAr (vV. 1, 14); TES (vV. 51, 3-6, 12-13); SPM (vV. 1-3, 13, 16); SPE (VV. 1-10, 13, 21-24)

3: 86; AU (vv. 2O, 31); CAr (vv. 1, 27); TES (vv. 8, 23-24)

4: 86; AU (vv. 2, 4, 5, 15, 17, 20)

5: 64 (vv. 16-end); 80 (vv. 14-17, 19-2O); 86; AU (vv. 3, 13-20); CAr (vv. 1, 18); TES

(vV. 2-5); FOR (vv. 2-5); SPM (vv. 3-5, 8-10, 19); SPE (vv. 1-5)

6: 64 (vv. 1-19); 80 (vv. 1-2); 84 (vv. 3, 12); 86; 251 (vv. 3-11); 262 (vv. 1-11); AU

(vv. 1-2, 6, 14); CAr (vv. 3, 15); S PM (vv. 3-13, 17-18); S PE (vv. 12-13, 19)

7: 86; 251 (vv. 24-end); AU (vv. 2, 8-11, 13-end); CAr (vv. 1, 12, 25); SPM (v. 25)

8: 32 (vv. 28-29, 39); 84 (vv. 3, 28); 86; 251 (vv. 1-5, 15-27); 271 (vv. 22-27); AU

(vv.1-17, 19-23, 26-30, 35, 38-39); CAr (vv. 15, 24, 31); TES (vv. 16-18, 24-25, 35-

37); F OR (vV. 16-18, 35-37); SPM (vv. 1-2, 9-11, 14, 31-32, 35-36); SPE (vv. 12-14,

$17,25,28,35-39)$

9: 86; AU (vv. 5, 11-22, 24-25, 27); CAr (vv. 1, 14, 22, 30); TES (vv. 3-5, 25-26); SPM (v. 5)

10: 31 (vv. 13-17); 84 (v. 8); 86 (vv. 1-2, 6); 87 (vv. 30-36); $25^{1}$ (vv. 15-17); AU (vv. 1, 8-10, 19); CAr (v. 5); S PE (vv. 10-13)

11: 67 (vv. 2-end); 79 (vv. 33-end); 84 (v. 13); 87 (vv. 1-16); AU (vv. 1, 11); CAr (vv. 1,

13, 25, 33, 36); TES (vv. 2O-21, 33-36); S PM (vv. 33-36); SPE (vv. 2O-22)

12: $67 ; 79$ (vv. 1-5, 17-end); 86 (vv. 13-16); AU (v. 10); CAr (v. 4); TES (vv. 14, 17, 19,

21); FOR (VV. 1-2); SPM (vV. 1-2, 10, 13-21); SPE (vv. 1-21)

13: $67 ; 79$ (vv. 1-5); 84 (v. 12); 86 (vv. 8-10); AU (vv. 1, 3-5, 8-11, 14); CAr (vv. 1, 6,

7); TES (VV. 3, 7-8); SPM (Vv. 1-8, 10, 13-14); SPE (Vv. 1-14)

14: 64 (vv. 10-end); 67; 79 (vv. 9-2o); 86 (vv. 8-10, 23); AU (vv. 1-6, 22); CAr (vv. 1,

$5,7,12)$; TES (Vv. 4, 17); SPM (Vv. 1, 16-21); SPE (VV. 1-25)

15:32 (vv. 9-13); 64 (vv.1-13); 67; 79 (vv. 3-13); 84 (v. 8); 86 (vv.11-end); 251 (vv.17-

29); AU (vv. 8-9, 16); CAr (vv. 4, 17); SPM (vv. 1-7, 13-14, 18-19, 26-27); SPE

(vv. 1-7, 26-27, 30)

16: 67 (vv. 1-6); 86; AU (vv. 17-18); CAr (v. 1); SPM (vv. 17-20); S PE (vv. 17-19) 


\section{Corinthians}

Complete: 51; 54; 58; 61; 75 (lac. 14:8-18); 76; 77; 78; 88; 89; AMst; PEL

Partial:

1: 64; 84 (v. 26); 86 (vv. 1-5); 251 (vv. 26-31); CAr (vv. 1, 4, 14, 31); TES (vv. 10, 17-24); SPM (VV. 20, 23-24, 26-29); S PE (vV. 10-13, 30-31)

2: $64 ; 84$ (v. 10); CAr (v. 1); SPM (vV. 7-8, 10-15)

3: 64 (vv. 1-5); 84 (vv. 1, 16); 251 (vv. 9-17); 271 (vv. 1-2, 7); CAr (vv. 1, 16); TES

(vv. 1-3, 16-2O); SPM (vv. 3, 16-2O); SPE (vv. 3-4, 16-19)

4: 271 (vV. 9-15); CAr (v. 9); TES (vV. 7, 20); SPM (v. 5); SPE (vV. 5-7, 11-14)

5: 84 (v. 6); 262 (vv. 6-13); CAr (v. 1); TES (vv. 7-8); SPM (vv. 6-8); SPE (vv. 1-13)

6: 64; 262 (vv. 1-11); CAr (v. 1); TES (vv. 1-2, 7-11, 15-2O); FOR (vv. 19-2O); SPM

(vV. 9-11, 15-2O); SPE (VV. 1-10, 15-2O)

7: $3^{2}$ (vv. 25-38); 64 (vv. 1-12, 19-26); 84 (v. 25); 251 (vv. 25-40); CAr (vv. 1, 12,

25); TES (VV. 1-7, 10-11, 29-34, 39-4O); S PM (Vv. 10-11, 25-26, 29-34, 38); SPE

(Vv. 1-40)

8: 84 (v. 4); CAr (v. 1); TES (vV. 2, 8); SPM (vV. 4-6); S PE (vV. 1-14, 7-13)

9: 84 (vv. 7, 24); 251 (vv. 7-15); 271 (vv. 7-17); CAr (vv. 1, 16); TES (vv. 24-25); FOR

(vv. 24-25); SPM (vv. 25-27); SPE (vv. 4-27)

10: 84 (v. 1); 87 (vv. 17-31); 251 (vv. 1-31); CAr (vv. 1, 14, 26); TES (vv. 1, 12-13, 22-23);

SPM (VV. 19-21, 31); SPE (Vv. 1, 5-14, 2O-33)

11: 84 (v. 23); 262 (vv. 20-34); CAr (vv. 1, 16, 27); TES (vv. 3, 19, 27, 33-34); FOR

(v. 3); SPE (vv. 1, 4-5, 17-22, 27-34)

12: 84 (v. 1); CAr (v. 4); S PM (vv. 3-11, 31); S PE (vV. 24-31)

13: 64 (vv. 13-end); CAr (v. 1); TES (vv. 2-8, 12); SPM (vv. 1-8, 13); S PE (vV. 1-8, 13) 14: 64 (vv. 1-5, 11-18); 84 (v. 4); CAr (vv. 2, 13, 26); TES (vv. 34-35); SPM (vv. 1, 12, 26); SPE (VV. 1, 12, 20, 26, 34-36)

15: $3^{2}$ (vv. 51-58); 64 (vv. 14-43); 84 (v. 1); 251 (vv. 1-28, 31-45, 47-58); CAr (vv. 1, 21, 39); TES (vv. 33, 36, 41-44, 47-49, 53-55); S PM (vv. 20-24, 33-46, 51-53); SPE (VV. 33-34, 58)

16: 64 (VV. 12-24); 411 (VV. 13-14); SPM (Vv. 13-14); S PE (Vv. 1-10, 13-14)

\section{Corinthians}

Complete: 51; 54; 58; 61; 75; 76; 77; 78; 88; 89; AMst; PEL

Partial:

1: 31 (vv. 19-22); 64; 67; 84 (v. 3); CAr (vv. 1, 3, 12, 23); SPM (vv. 2-3, 21-22); SPE (v. 12)

2: 64 (vv. 1-10); 67; CAr (v. 12); SPM (vV. 5-11); SPE (vv. 5-11) 
3: 64 (vv. 17-end); 67; CAr (v. 7); TES (vv. 14-16); SPM (vv. 17-18)

4: 64; 67; 84 (v. 6); 271 (vv. 5-18); CAr (vv. 3, 16); SPM (vv. 1-4, 8-11); SPE (vv. 1-2,

$5,7-13,16-18)$

5: 32 (vv. 11-20); 64 (vv. 1-12, 14-end); 67; CAr (v. 11); TES (v. 10); 271 (v. 1); FOR

(v. 15); SPM (Vv. 1-5)

6: $3^{2}$ (Vv. 1-10, 14-18); 64 (Vv. 1-3); $67 ; 84$ (Vv. 2, 12); 87 (Vv. 2-10, 12-18); $25^{1}$ (Vv. 2-

15); CAr (v. 2); TES (v. 14); SPE (vv. 1-10, 13-18)

7: $3^{2}$ (v. 1); 64 (vv. 10-end); 67 (vv. 1-4); 84 (v. 4); CAr (vv. 1, 12); SPM (vv. 9-10);

SPE (VV. 8-11)

8: 64 (vv. 1-12); CAr (vv. 9, 22); TES (vv. 12-15); SPM (v. 21); SPE (vv. 1-21)

9: 64 (vv. 10-end); 84 (v. 6); CAr (v. 10); TES (vv. 6-7, 9-12); SPM (vv. 5-7); SPE

(vv. 1-15)

10: $64 ; 84$ (v. 15); 87 (vv. 7-14); CAr (v. 7); SPM (vv. 3-5, 7); SPE (vv. 17-18)

11: 64 (vv. 1-21); 83 (vv. 33-end); 84 (v. 19); CAr (v. 1); SPM (vv. 12-15); SPE (vv. 23-

12: 64 (vv. 14-end); 67 (vv. 18-end); 83 (vv. 1-14); CAr (vv. 1, 14); TES (vv. 7-9);

FOR (V. 2); S PE (VV. 9-1O, 14-15, 2O-21)

13: 64 (Vv. 1-10); 411 (v. 11); CAr (v. 7); SPM (v. 13); SPE (vv. 7, 11)

\section{Galatians}

Complete: 51; 54; 58; 61; 75; 76; 77; 78; 88; 89; 135; MAR (lac. 3:11-19; 5:18-6:1); AMst; HI; AU; PEL; THr

\section{Partial:}

1: 67; 262 (vv. 3-12); CAr (vv. 1, 6); TES (v. 10); SPE (v. 10)

2: 64 (vv. 5-end); $67 ; 84$ (v. 9); CAr (vv. 6, 14)

3: 64; 67 (vv. 1-29); 84 (vv. 11, 27); 87 (vv. 24-end); CAr (vv. 1, 24); TES (vv. 6-9); SPM (Vv. 3, 19-20)

4: 64 (vv. 1-3, 6-end); 84 (vv. 1, 12); 87 (vv. 1-7); CAr (v. 19); TES (v. 4); SPM (vv. 4$6,8-12)$

5: 64 (vv. 1-2); 251 (vv. 13-26); CAr (vv. 10, 14); TES (vv. 14-15, 17, 19-24); SPE (vv. 6, $13-26)$

6: 64 (vv. 5-18); 84 (v. 7); 251 (vv. 1-2, 7-14); CAr (vv. 1, 8); TES (vv. 1-2, 14); SPM (vV. 7-10); SPE (VV. 1-10) 